\title{
DISCLOSE : DISsection of CLusters Obtained by SEries of transcriptome data using functional annotations and putative transcription factor binding sites
}

\author{
Evert-Jan Blom ${ }^{1}$, Sacha AFT van Hijum¹,2,3, Klaas J Hofstede1, Remko Silvis ${ }^{1}$, \\ Jos BTM Roerdink ${ }^{4}$ and Oscar P Kuipers*1
}

\begin{abstract}
Address: ${ }^{1}$ Molecular Genetics, Groningen Biomolecular Sciences and Biotechnology Institute, University of Groningen, The Netherlands, 2Interfacultary Institute for Genetics and Functional Genomics, Ernst-Moritz-Arndt-University, Friedrich-Ludwig-Jahnstraße, 15A 17487, Greifswald 17489, Germany, ${ }^{3}$ NIZO Food Research, PO Box 20, 6710 BA Ede, the Netherlands and ${ }^{4}$ Institute for Mathematics and Computing Science, University of Groningen, Nijenborgh 9, 9747 AG, Groningen, The Netherlands

Email: Evert-Jan Blom - e.j.blom@rug.nl; Sacha AFT van Hijum - sacha.vanhijum@nizo.nl; Klaas J Hofstede - klaasjanhofstede@home.nl; Remko Silvis - secutortheone@gmail.com; Jos BTM Roerdink - j.b.t.m.roerdink@rug.nl; Oscar P Kuipers* - o.p.kuipers@rug.nl

* Corresponding author
\end{abstract}

Published: 16 December 2008

BMC Bioinformatics 2008, 9:535 doi:10.1186/147/-2105-9-535
Received: 25 April 2008

Accepted: 16 December 2008

This article is available from: http://www.biomedcentral.com//47I-2105/9/535

(c) 2008 Blom et al; licensee BioMed Central Ltd.

This is an Open Access article distributed under the terms of the Creative Commons Attribution License (http://creativecommons.org/licenses/by/2.0), which permits unrestricted use, distribution, and reproduction in any medium, provided the original work is properly cited.

\begin{abstract}
Background: A typical step in the analysis of gene expression data is the determination of clusters of genes that exhibit similar expression patterns. Researchers are confronted with the seemingly arbitrary choice between numerous algorithms to perform cluster analysis.

Results: We developed an exploratory application that benchmarks the results of clustering methods using functional annotations. In addition, a de novo DNA motif discovery algorithm is integrated in our program which identifies overrepresented DNA binding sites in the upstream DNA sequences of genes from the clusters that are indicative of sites of transcriptional control. The performance of our program was evaluated by comparing the original results of a time course experiment with the findings of our application.

Conclusion: DISCLOSE assists researchers in the prokaryotic research community in systematically evaluating results of the application of a range of clustering algorithms to transcriptome data. Different performance measures allow to quickly and comprehensively determine the best suited clustering approach for a given dataset.
\end{abstract}

\section{Background}

DNA microarray technology is commonly used to study mRNA expression levels of genes under different experimental conditions. Clustering approaches are widely used in the analysis of gene expression data. The ability to identify groups of genes exhibiting similar expression patterns by clustering allows for detailed biological insights into global regulation of gene expression and cellular proc- esses. Clustering methodology is considered a potent means to infer putative gene function $[1,2]$.

In the process of the analysis of transcriptome data, researchers are often faced with the choice between a wide variety of clustering methods and associated parameters. The results of the application of different clustering algorithms to the same dataset will place genes in different 
clusters and therefore result in different biological interpretations of the same dataset. Moreover, selecting the most appropriate clustering method and parameters heavily depends on the experience of the researcher and on the nature of the dataset analyzed.

Several studies have shown the relevance of applying external measures (i.e., using prior biological knowledge) to more objectively evaluate the results of clustering algorithms ([3-6]). Central in this approach is the assumption that genes involved in similar biological processes are more likely to be co-transcribed. Therefore, selecting a clustering method the clusters of which are most enriched with biological processes is considered as a relevant starting point for the biological interpretation of a DNA microarray dataset [6-9].

Co-clustered genes may also represent a candidate set of coregulated genes, i.e., genes of which the expression is regulated by the same transcription factor. The discovery of putative regulatory motifs in cis-regulatory regions of genes that are part of the same cluster could therefore allow identification of new TF targets [10]. Existing implementations that employ motif discovery on clusters obtained by DNA microarray $[7,8,11]$ leave the downstream analysis of the motifs to be performed by the researcher. More importantly, no feedback concerning the results of the analysis is presented for the used clustering algorithm and associated parameters, making it difficult to compare the effect on the results of different clustering parameters or methods to the same dataset. Ideally, quantitative information concerning the functional and motif enrichments of the tested clusters should be provided after each clustering analysis. This information would then allow for a more objective selection of optimal clustering parameters based on biological criteria. Lastly, all available software packages are not specifically suited for prokaryotic data analysis since they do not support prokaryote-specific data sources (e.g., operons, specific genome annotations).

We have developed the application DISCLOSE for prokaryotes that benchmarks clustering methods using biological annotations and the SCOPE DNA binding site detection algorithm [12]. This algorithm allows the prediction of cis-regulatory motifs of genes which are part of the same cluster. In addition, additional occurrences of identified motifs are determined. Moreover, putative motifs are compared with known DNA binding sites as well as a functional analysis of the genes bearing the motif in their upstream region.

\section{Program overview}

The DISCLOSE application allows for an automated scoring based on different criteria of the different clusters in each clustering analysis. This scoring is followed by a decision by the researcher on the most suitable clustering method for the dataset analyzed based on one metric. Various metrics (see below) are available to assess the results of the clustering analysis. Each metric provides for a unique measure to filter the results of a clustering analysis and can therefore be used to address different research questions; e.g., selection of a clustering analysis which yields a large number of overrepresented motifs or a clustering analysis which produces a large number of significant overrepresented metabolic pathways. Based on the chosen clustering analysis, DISCLOSE provides an indepth analysis of clustering results together with an intuitive visualization.

\section{I Input}

A process overview of DISCLOSE is shown in Figure 1. The input data for DISCLOSE consists of transcriptome data (Fig. 1A) and genome files (e.g., EMBL or Genbank). DISCLOSE supports a broad variety of prokaryotic gene identifiers, including locus tags and gene names.

The DNA binding site detection algorithm uses operon information and the genomic sequence in FASTA format (see Fig. 1G). However, DISCLOSE also supports a single gene-based analysis if no operon information is available. Moreover, known binding site information can be used to evaluate the results of the DNA binding site detection algorithm.

\subsection{Processing}

\subsection{Clustering of gene expression data}

Two widely used clustering algorithms (K-means and Self Organizing Maps) from the TIGR Multiexperiment Viewer (MeV) package http://www.tm4.org/mev.html are implemented in DISCLOSE (Fig. 1B). The application of different parameter settings to a clustering analysis is facilitated by allowing for a parameter range and/or different correlation measures for each clustering approach, e.g., a Kmeans clustering with five to twenty clusters using Euclidean and Pearson correlation measures (Fig. 1C).

\subsubsection{Evaluation of external clustering applications}

The two most used clustering algorithms have been implemented in DISCLOSE. Since many other clustering methods exist, we have chosen to allow the evaluation of results obtained from external clustering applications in DISCLOSE (Fig. 1D).

\subsubsection{Evaluating clusters using biological knowledge}

The identification of significantly enriched categories in a cluster of co-expressed genes enables users to focus on relevant biological phenomena. Assuming a normal distribution of the number of genes from the clusters for each functional category, one expects a difference in the pro- 


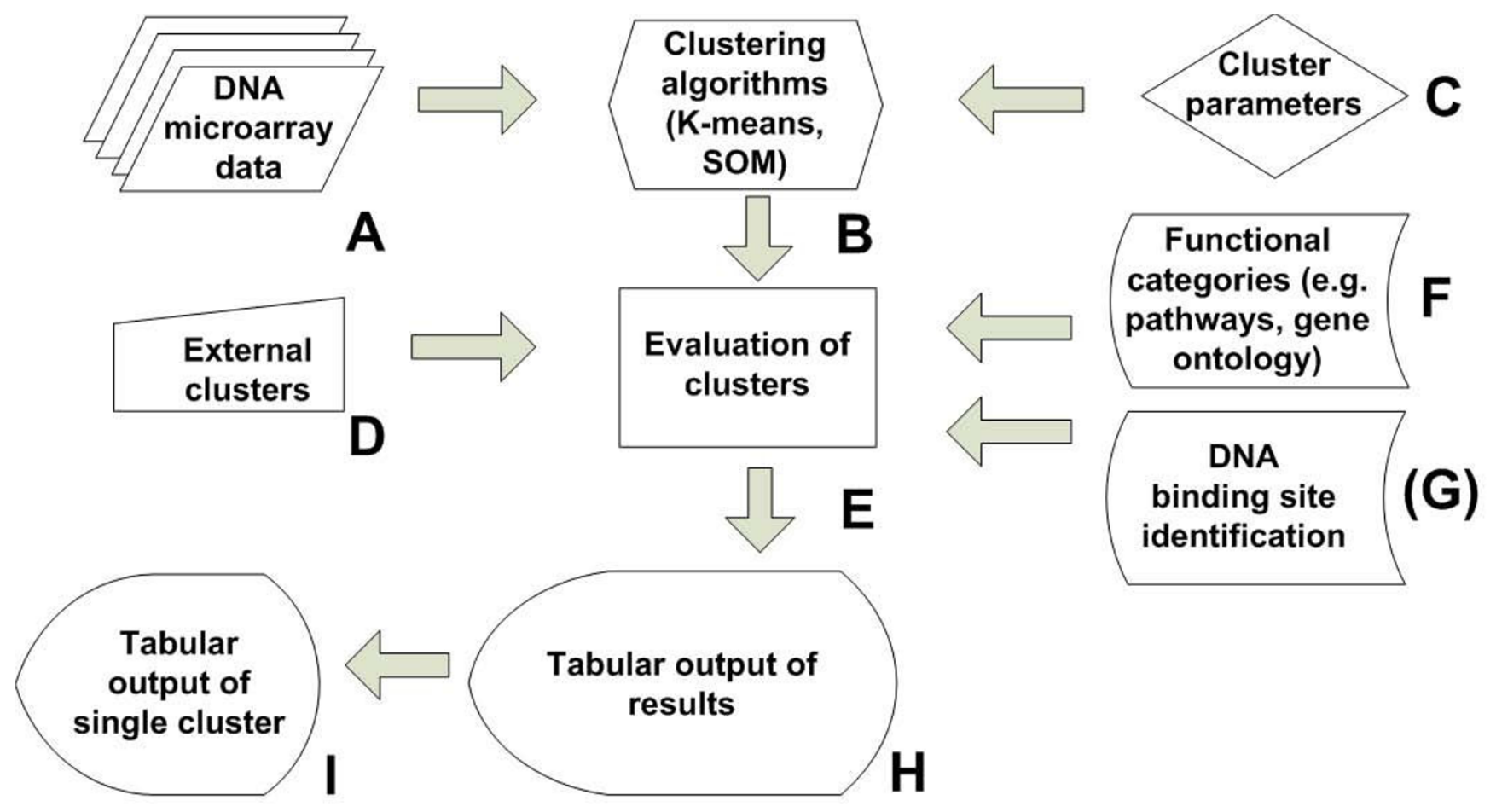

Figure I

Flow diagram. The DISCLOSE application uses functional categories to evaluate the cluster results given a dataset (A), clustering algorithms (B), and clustering parameters (C). Clustering can be performed by the DISCLOSE application or based on results from external clustering programs $(D)$. Each clustering run is evaluated $(E)$ for overrepresented functional categories by the program using different annotation sources $(F)$, and optionally by a motif identification algorithm $(G)$. Lastly, results of the clustering analysis are cumulated in a tabular display in which each row shows the summary of the application of a clustering method to the data $(\mathrm{H})$. From the tabular display, selecting results for an individual clustering (I) allows for a cluster based analysis (see Fig. 2A).

portion of genes for a category present in each cluster compared to the genes from a reference set (e.g., the remaining genes from the studied organism). To identify clusters that contain a significantly enriched number of genes from a certain functional class, the distribution of genes from a gene set (e.g., a cluster of genes) is compared to the genes in the reference set (e.g., the remainder of the genes in all other clusters).

A hypergeometric distribution test is used to calculate $p$ values for each functional category from each cluster. This $p$-value describes the probability of observing an enrichment of genes from a functional category in a cluster by chance (Fig. 1E). The number of false-positives for the initial cluster evaluation (Fig. 1E) is controlled by a strict Bonferroni multiple testing correction (taking into account the clustering runs), while additional corrections ([13]) are used upon detailed analysis of selected results.

\subsubsection{De novo identification of DNA binding sites}

Clustering algorithms allow for the identification of groups of genes that exhibit similar expression patterns. This co-expression could be explained by transcriptional co-regulation. Identification of overrepresented DNA binding sites in genes of the same cluster is performed by the SCOPE method [12]. This method utilizes three specialized algorithms; BEAM for non-degenerate motifs, PRISM for degenerate motifs and SPACER for bipartite motifs. Key aspects of SCOPE are high sensitivity and specificity for a broad range of motifs (i.e., perfect, degenerated and gapped motifs), requirement of a minimum of parameters for motif detection, and speed [12].

\subsubsection{Characterization of putative motifs}

For various model organisms reference databases on transcriptional regulation have been created that summarize experimentally characterized transcription factors, their 
binding sites and the genes they regulate (e.g., from DBTBS [14] for Bacillus subtilis or regulonDB [15] for Escherichia coli). The binding sites derived from these databases can be incorporated in DISCLOSE, allowing for a comparison of known binding sites to putative DNA binding sites found by the SCOPE algorithm. This feature allows researchers to distinguish between known and unknown binding sites. In addition, aligned putative motif instances identified by the SCOPE algorithm are used to create position specific scoring matrices. These matrices are subsequently used to score the upstream and coding DNA sequences from all genes of the studied organism. The prioritized results of this analysis allow researchers to identify additional genes that do contain the putative motif but were not part of the original cluster.

Lastly, DISCLOSE attempts to functionally characterize motifs by identifying significantly enriched categories using the genes that contain the motif. This analysis is different as compared to a standard functional enrichment analysis. Since the motif analysis only uses operon information, the enrichment of categories is calculated by taking into account the operons instead of genes. This analysis yields a $p$-value which describes the probability of observing an enrichment of operons belonging to a specific functional category in the operon members bearing the motif by chance. The results of this examination yield insights concerning the biological processes that could be controlled by the putative motif.

\subsection{Output}

Quantitative results of over representations for each clustering evaluation (e.g., a K-means clustering for 20 clusters using a Euclidean correlation measure) are represented in a tabulated view (Fig. 1I). A single row in this view includes information from an individual clustering run for the following metrics:

1. Number of significant overrepresented functional categories from each annotation source (e.g., the total number of overrepresented metabolic pathways).

2. Total number of significant overrepresented functional categories from all annotation sources (e.g., all overrepresented metabolic pathways, GO categories etc).

3. The number of clusters which are enriched for one or more functional categories.

4. The score of the most overrepresented DNA binding site.

5. The number of overrepresented DNA binding sites that exceed a predefined threshold.
6. Number of functional categories from one annotation source that were found overrepresented in gene members of a cluster that contain a certain motif in their upstream region.

7. Number of functional categories for all annotation sources that were found overrepresented in gene members of a cluster that contain a certain motif in their upstream region.

Several filtering options are available for each of the described metrics. Each metric provides for a unique measure for users to select the highest scoring clustering results based on different criteria, e.g., a clustering that yields the highest number of overrepresented metabolic pathways or the most significantly overrepresented DNA binding sites. This allows researchers to select the most optimal clustering run for their research question. Graphical representations of the results are available for different stages in the analysis which will be discussed in the upcoming sections.

\subsection{Complete results analysis}

In addition to saving the complete contents of the tabulated view to a HTML file, a robustness analysis is performed on all of the clustering runs. This analysis determines the frequency of occurrence for every functional category (e.g., a robustness frequency for a functional category of $50 \%$ indicates that it is significantly overrepresented in individual clusters from $50 \%$ of the clustering runs). Robust functional categories are a good starting point for an analysis since they occur in relatively large fractions of the clustering results. Less robust functional categories allow for the analysis of functional categories that would have been missed using more general clustering parameters.

\subsubsection{Functional analysis for individual clustering runs}

Based on the results of a single clustering run, a graphical representation (Fig. 2A) of the overrepresented categories is generated by FIVA [13]. Individual cluster information from a single clustering run is available, which enables a focused analysis of individual clusters.

\subsubsection{Visualization of putative DNA binding sites for individual clustering runs}

In addition to the functional analysis described in the previous section, DISCLOSE is capable of identifying overrepresented DNA binding sites in clusters of co-expressed genes. The putative DNA binding sites that are identified from the SCOPE algorithm are visualized as sequence logos [16] and displayed in HTML files (Fig. 2A to Fig 2F). In addition, putative motifs are compared with known DNA binding site information (e.g., from DBTBS [14] or regulonDB [15], see Fig. 2F). The results of this compari- 


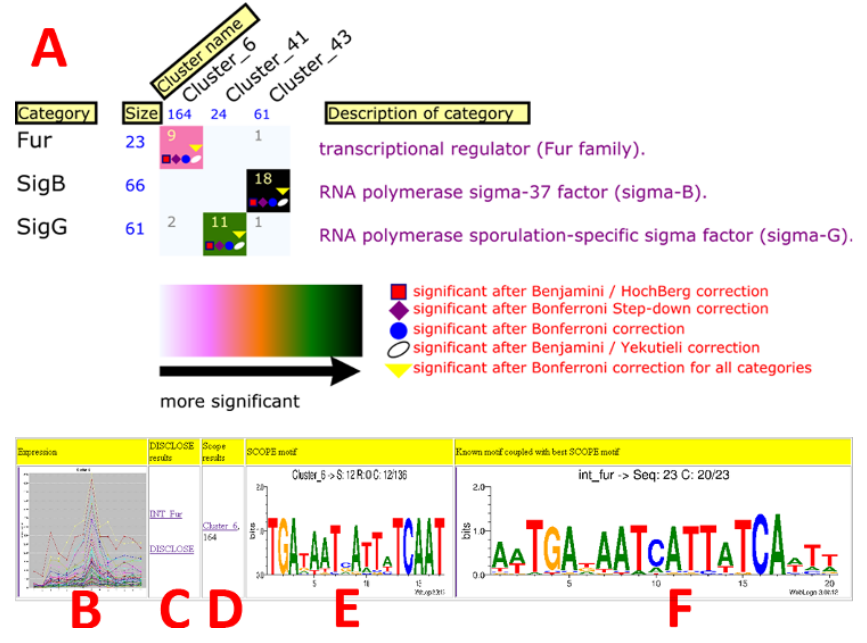

Figure 2

Visualization. A. Genes from the DNA microarray data were clustered. The size of each cluster is displayed in blue underneath the cluster name. Numbers in each colored rectangle represent absolute values of occurrences. The significance of the overrepresentation is visualized in a colour gradient which is displayed at the bottom of the plot. The description of each category is placed at the right. Multiple testing correction results are visualized using five different symbols to distinguish between the individual corrections. The number of symbols placed in each rectangle corresponds to the number of multiple testing corrections after which the annotation is found significant (see [13] for more details concerning this visualization). The graphical representation of the overrepresented DNA binding sites from the SCOPE algorithm consists of several components. The results of SCOPE based on a single cluster are discussed: $\mathrm{B}$. The expression graph of the genes in the cluster. C. Contains information concerning overrepresented functional categories and a link to the results of DISCLOSE. D. Link to the results of SCOPE. E. The highest scoring motif found in the cluster. F. The highest scoring motif is compared with existing binding site information. The known motif that matches the putative motif best is displayed.

son are integrated in the HTML files. Detailed information for every putative motif is available, showing identified functional enrichments of in the operon members of the motif and the raw output of the SCOPE algorithm in text files. Moreover, information concerning additional occurrences of the putative motif in the upstream or coding regions from other operons of the genome is integrated in the HTML files. Lastly, an operon visualization is created which show the genomic context for all known and putative motifs to facilitate the motif analysis (Fig 3).

\section{Results and discussion}

Our DISCLOSE application was used to identify significant functional categories and DNA binding sites. To eval-

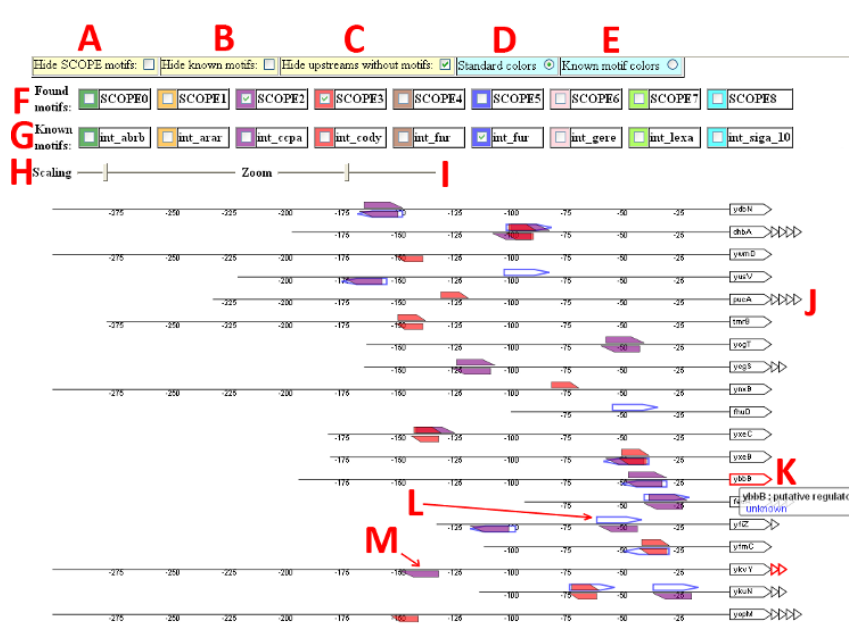

Figure 3

A customizable graphical representation of DNA binding sites. The Scalable Vector Graphics visualization displays the genomic context of putative and known motifs in the upstream sequences of the operons. The user interface allows users to interact with the visualization. A) Hide de novo motifs. B) Hide known motifs from literature. C). Hide upstream regions without any putative or known motifs. D). Use standard coloring of putative motifs. E). Use coloring of putative motifs based on best hit with known motif. F). Every found motif can be displayed or hidden from the visualization using checkboxes. G) Known motifs can be displayed or hidden from the visualization using checkboxes. $H$ ). The scaling slider adjusts the width between the upstream sequences. I). The zooming slider allows for zooming of the visualization. J). The first structural gene of each operon is a large polygon, whilst the other genes are represented using smaller polygons. K). Genes coding for a putative regulator are colored red. Hovering with the mouse over the genes creates a tooltip displaying the function of the gene. L). Open polygons represent known binding sites derived from literature sources. M). Filled polygons depict putative motifs.

uate the performance of our program, we compared the original clustering analysis results (see Table 1) of a time course DNA microarray experiment of B. subtilis ([17]) with the findings of DISCLOSE. With this time-course experiments the authors explore the transcriptional changes that occur during germination and outgrowth of B. subtilis spores. The highest scoring categories from our DISCLOSE analysis, i.e., categories with a significance frequency above 10\%, are listed in Table 2. Overlapping redundant categories that represent similar functional groupings were removed from the table. The majority of the significantly overrepresented categories that were identified by our analysis recapitulated the original results.

Furthermore, additional biological phenomena were identified by DISCLOSE that were not discussed by the 
Table I: Biological phenomena discussed in the original article

\begin{tabular}{|c|c|c|}
\hline Functional category & DISCLOSE & Significance frequency \\
\hline Purine biosynthesis & $x$ & $91 \%$ \\
\hline Cell growth & $x$ & $88 \%$ \\
\hline General stress response & $x$ & $88 \%$ \\
\hline tricarboxylic acid cycle & $x$ & $86 \%$ \\
\hline Sigma $D$ regulon (motility) & $x$ & $85 \%$ \\
\hline Glycolysis & $x$ & $72 \%$ \\
\hline cell division & $x$ & $71 \%$ \\
\hline pyrimidine biosynthesis & $x$ & $70 \%$ \\
\hline DNA replication and DNA repair functions & $x$ & $66 \%$ \\
\hline Sulfur amino acid metabolism & $x$ & $19 \%$ \\
\hline aspartate metabolism & $x$ & $16 \%$ \\
\hline serine metabolism & $x$ & $12 \%$ \\
\hline fatty acid biosynthesis & $x$ & $12 \%$ \\
\hline drug transporter activity & $x$ & $3.1 \%$ \\
\hline $\mathrm{Na}+/ \mathrm{H}+$ antiporters & $x$ & $0.6 \%$ \\
\hline RNA modification & $x$ & $0.3 \%$ \\
\hline Multidrug transporters & - & \\
\hline
\end{tabular}

The original analysis of the study of Keyser et al [17] revealed several biological phenomena that are found to be induced during the DNA timecourse experiment. The described biological phenomena were matched with the results of the robustness analysis (complete analysis is listed in Table 2). Phenomena discussed in the original analysis are listed in the first column. A match with the results of DISCLOSE is indicated in the second column. Information concerning the significance frequency is shown in column three.

authors (Table 2). However, some categories that were described in the original study did not meet the $10 \%$ threshold that was used in our analysis. These reported categories were not found in the original analysis using a clustering based analysis but using an analysis which has been performed on the highest expressed genes from every individual time point.

\section{DNA binding site analysis of DISCLOSE}

For the overrepresented DNA binding site analysis of the data of Keijser et al, we selected the clustering run that yielded the highest number of overrepresented motifs. The combined visualization of known and putative motifs allowed for a rapid determination of genes with instances of motifs that matched to a known DNA binding site (see
Additional file 1). A total number of 12 putative motifs that were identified by DISCLOSE matched one of the 45 motifs that are described in DBTBS. Additionally, 2 motifs were linked to known motif binding sites based on literature information. Furthermore, DISCLOSE also discovered a number of motifs that were not described before (Fig 4).

\section{Discussion of the results}

The results of our DISCLOSE analysis show that we were able to identify most of the functional overrepresented categories that are discussed in the original study. However, a number of functional categories were not recovered using our approach due to the nature of the analysis employed in the original study which was based on anal- 
Table 2: Results of robustness analysis of DISCLOSE

\begin{tabular}{|c|c|c|c|}
\hline Functional category & Member size & Significance frequency & In original study \\
\hline GO-0006164 : purine nucleotide biosynthetic process & 28 & $94.03 \%$ & Y \\
\hline COG-F : Nucleotide transport and metabolism & 84 & $93.56 \%$ & Y \\
\hline GO-0003735 : structural constituent of ribosome & 59 & $88.06 \%$ & Y \\
\hline INT-SigB : general stress sigma factor & 66 & $87.91 \%$ & Y \\
\hline COG-J : Translation, ribosomal structure and biogenesis & 161 & $87.12 \%$ & Y \\
\hline PW-path-bsu00020: Citrate cycle & 18 & $86.18 \%$ & $\mathrm{~N}$ \\
\hline GO-0003723 : RNA binding & 107 & $85.87 \%$ & Y \\
\hline COG-N : Cell motility and secretion & 57 & $85.71 \%$ & Y \\
\hline INT-SigG : late forespore-specific gene expression & 61 & $84.30 \%$ & $\mathrm{~N}$ \\
\hline PW-path-bsu00193: ATP synthesis & 8 & $83.04 \%$ & Y \\
\hline UP-67 : Ligase & 78 & $79.74 \%$ & Y \\
\hline GO-0006935: chemotaxis & 26 & $73.46 \%$ & Y \\
\hline UP-56: Glycolysis & 19 & $72.21 \%$ & Y \\
\hline UP-29 : Cell division & 32 & $71.11 \%$ & Y \\
\hline PW-path-bsu00720: Reductive carboxylate cycle & 13 & $70.32 \%$ & $\mathrm{~N}$ \\
\hline PW-path-bsu00240 : Pyrimidine metabolism & 51 & $68.60 \%$ & Y \\
\hline PW-path-bsu00970: Aminoacyl-tRNA biosynthesis & 23 & $68.28 \%$ & Y \\
\hline COG-L : DNA replication, recombination and repair & 138 & $65.93 \%$ & Y \\
\hline UP-I5: Threonine biosynthesis & 3 & $58.55 \%$ & $\mathrm{~N}$ \\
\hline COG-G : Carbohydrate transport and metabolism & 246 & $55.88 \%$ & Y \\
\hline GO-0006520 : amino acid metabolic process & 184 & $53.53 \%$ & Y \\
\hline UP-I24 : Sporulation & 180 & $49.92 \%$ & Y \\
\hline INT-SigK : late mother cell-specific gene expression & 57 & $47.40 \%$ & $\mathrm{~N}$ \\
\hline PW-path-bsu03070 : Type III secretion system & 9 & $44.27 \%$ & $\mathrm{~N}$ \\
\hline INT-PurR : negative regulation of the purine operons & 10 & $43.32 \%$ & $\mathrm{~N}$ \\
\hline GO-00I5293 : symporter activity & 82 & $35.63 \%$ & Y \\
\hline UP-25 : Porphyrin biosynthesis & 13 & $27.62 \%$ & $\mathrm{~N}$ \\
\hline UP-I79: Folate biosynthesis & 6 & $27.31 \%$ & $\mathrm{~N}$ \\
\hline
\end{tabular}


Table 2: Results of robustness analysis of DISCLOSE (Continued)

\begin{tabular}{|c|c|c|c|}
\hline PW-path-bsu00190: Oxidative phosphorylation & 31 & $26.05 \%$ & $\mathrm{~N}$ \\
\hline PW-path-bsu02060 : Phosphotransferase system (PTS) & 27 & $24.96 \%$ & $\mathrm{~N}$ \\
\hline COG-D : Cell division and chromosome partitioning & 33 & $24.64 \%$ & Y \\
\hline GO-0008360 : regulation of cell shape & 36 & $24.17 \%$ & Y \\
\hline PW-path-bsu00740 : Ribo avin metabolism & 5 & $23.54 \%$ & $\mathrm{~N}$ \\
\hline PW-path-bsu00030 : Pentose phosphate pathway & 24 & $23.39 \%$ & $\mathrm{~N}$ \\
\hline GO-0009086 : methionine biosynthetic process & 15 & $22.76 \%$ & Y \\
\hline UP-I7 : Hydrogen ion transport & 15 & $21.66 \%$ & Y \\
\hline INT-SigE : early mother cell-specific gene expression & 82 & $21.66 \%$ & $\mathrm{~N}$ \\
\hline PW-path-bsu00920 : Sulfur metabolism & 15 & $19.62 \%$ & $\mathrm{~N}$ \\
\hline INT-SigA : RNA polymerase major sigma-43 factor & 320 & $18.52 \%$ & $\mathrm{~N}$ \\
\hline COG-O : Posttranslational modification, protein turnover, chaperones & 98 & $17.11 \%$ & $\mathrm{~N}$ \\
\hline PW-path-bsu00400 : Phenylalanine, tyrosine and tryptophan biosynthesis & 28 & $16.16 \%$ & $\mathrm{~N}$ \\
\hline PW-path-bsu00252: Alanine and aspartate metabolism & 21 & $16.01 \%$ & Y \\
\hline GO-0009252 : peptidoglycan biosynthetic process & 32 & $15.22 \%$ & Y \\
\hline PW-path-bsu00260 : Glycine, serine and threonine metabolism & 34 & $12.55 \%$ & Y \\
\hline UP-84 : Fatty acid biosynthesis & 11 & $12.55 \%$ & Y \\
\hline GO-0000103: sulfate assimilation & 7 & $10.67 \%$ & $\mathrm{~N}$ \\
\hline GO-0000I05: histidine biosynthetic process & II & $10.36 \%$ & $\mathrm{~N}$ \\
\hline PW-path-bsu00670: One carbon pool by folate & II & $10.20 \%$ & $\mathrm{~N}$ \\
\hline
\end{tabular}

The performance of DISCLOSE was evaluated by comparing the clustering analysis results of a time course DNA microarray experiment of Bacillus subtilis ([I7]) with the results obtained by DISCLOSE. This analysis recapitulated most of the findings of the original study. In addition, several significantly overrepresented categories were found by DISCLOSE that were not discussed by the authors. The following table list the functional categories that are identified by DISCLOSE from the robustness analysis with a significance frequency of at least $10 \%$. The last column indicates if a category was found significant in the original study. Redundant functional categories were removed from the table (e.g., functional category GO:0006096-glycolysis was not listed in the table since this functional category was already covered by a UniProt category UP:56-glycolysis). (Abbreviations used; GO : Gene Ontology, INT : Regulon, PW: metabolic pathway, UP : UniProt and COG : Clusters of Orthologous Gtroups)

ysis of differentially expressed genes in single time points. The single-time point analysis is tedious and does not take into account the temporal properties of the dataset.

The interactive visualization module for overrepresented motifs by DISCLOSE facilitated the detection of putative motifs as well as the discovery of motifs that have been described in literature.

\section{Conclusion}

Choosing a clustering method and associated parameters for a given DNA microarray dataset is a challenging task. Moreover, commonly used clustering algorithms lack the ability to annotate the clusters using functional information. This information is crucial to comprehend the underlying biology of the experiment. Here, we present DISCLOSE, an exploratory application that benchmarks clustering methods using functional annotations and a de 


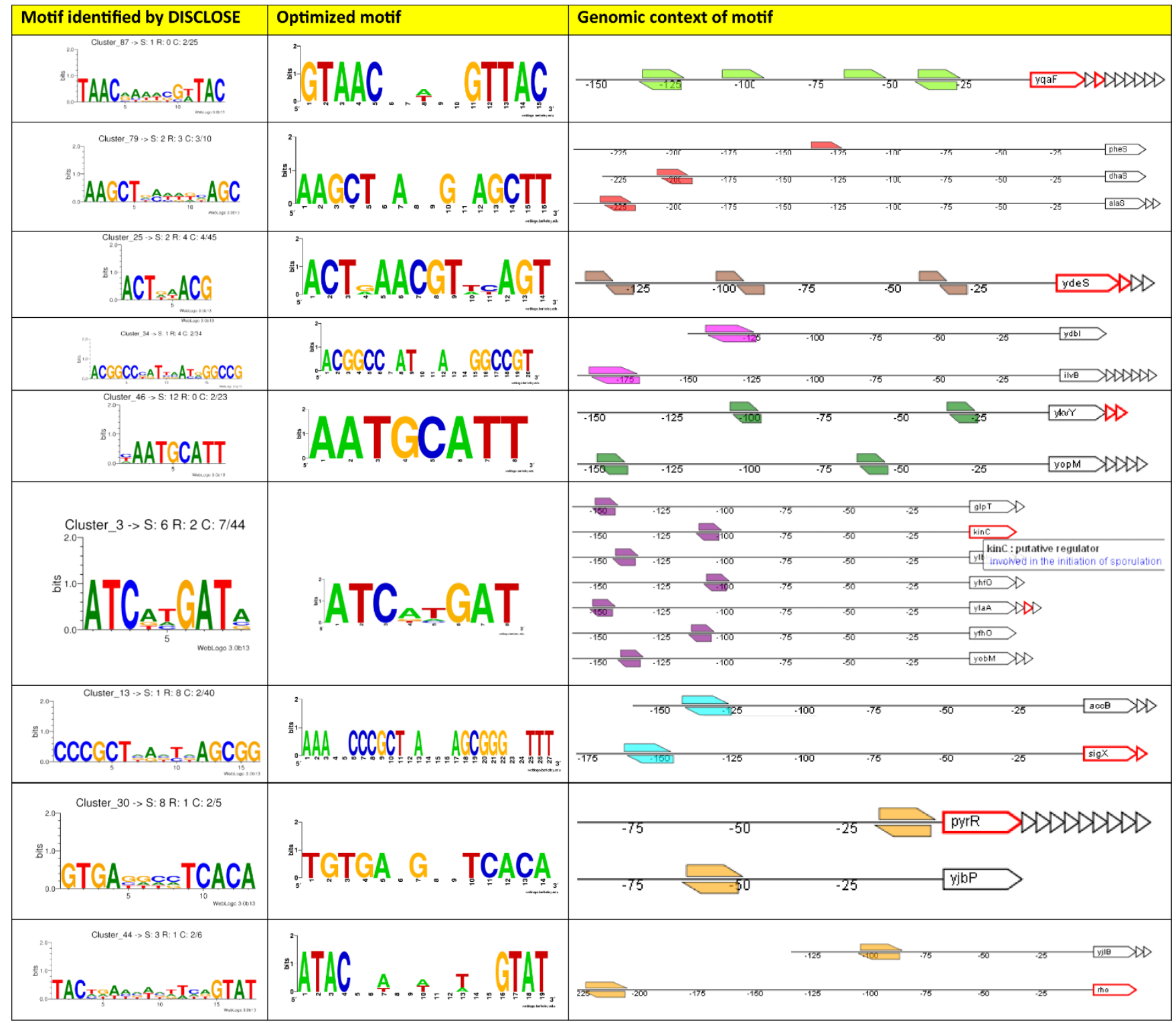

Figure 4

Non-validated results of overrepresented DNA binding sites. DISCLOSE was also able to detect several motifs in clusters that could not be matched with motifs from literature. The motifs identified by DISCLOSE are visualized as sequence logos [16] and are displayed in the first column. An optimized version of the motif is placed in the second column whilst the genomic context of the instances are displayed in column three.

novo motif discovery algorithm. DISCLOSE allows to select the most appropriate clustering method and to visually inspect the clusters obtained for a given DNA microarray dataset. Our application quantitatively describes the most stable overrepresented functional categories in the clusters. This methodology allows for a more objective and complete interpretation of the dataset analyzed. Our application offers the following advantages to existing tools:
- benchmarks clustering methods using enrichment analysis and motif discovery

- supports K-means and SOM clustering algorithms

- robustness analysis for functional categories

- ready-to-use databases for over 600 prokaryotic organisms 
- functional enrichment analysis of putative motifs

- identification of additional motif occurrences

- matching of putative motifs with known motifs

- interactive visualization of genomic context of known and putative motifs.

- stand-alone application (supports all major operating systems)

\section{Methods}

\section{I Software package}

DISCLOSE was programmed as a standalone application in Java using the Eclipse http://www.eclipse.org/ framework and it runs on all Java-supporting operating systems (Windows, Linux and Mac OSX). The graphical output can be viewed by all web browsers that are able to process Scalable Vector Graphics.

DISCLOSE features the following annotation modules: $\mathrm{i}$. Gene Ontology, ii. Metabolic pathways (KEGG), iii. COG classes, iv. Regulatory interactions, v. UniProt keywords vi. and user-defined functional categories (Fig. 1F). The supplementary materials contain ready-to-use databases for over 600 prokaryotic organisms. The software package contains in addition a manual and an example analysis using a publicly available dataset.

\section{Dataset used for validation}

The dataset is part of a transcriptome analysis from a study on the growth transitions of Bacillus subtilis. Data from this experiment was obtained from the Gene Expression Omnibus database from NCBI [18] (accession number: GSE6865). The authors of the original study applied a Kmeans clustering to reveal patterns of temporal gene expression. The optimum number of clusters was revealed by principal-component analysis and ordered by the timing of expression. A detailed analysis based on individual time points was conducted by JProGO [19] to identify overrepresented groups of functionally related genes. From this analysis, the authors have selected several functional categories from a list of significantly overrepresented categories (see Table 1).

\section{DISCLOSE analysis}

Our analysis was conducted using a K-means clustering using a range from 10 to 100 clusters for all correlation measures. For each cluster that was analyzed the DNA binding site reported the 10 most overrepresented motifs. Finally, the results of a clustering run that yielded the highest number of motifs with a score above 15 together with a robustness analysis with a $10 \%$ cut off for all clusters were analyzed.

\section{Gene annotations}

A genome file for Bacillus subtilis was obtained from NCBI and supplemented with $\mathrm{db}_{\text {_x }}$ refield information from an EMBL genome review file from EBI [20]. COG information from a local whog file [21] was loaded by using the organism abbreviation: bsu. Pathway information was obtained from KEGG [22]. The latest Gene Ontology (GO) obo file was used from the Gene Ontology website [23]. Functional categories based on Uniprot keywords [24] were imported as well as information from the DBTBS database [14] for the interaction annotation module.

\section{Availability and requirements}

- Project name: DISCLOSE

- Project homepage: http://bioinformatics.biol.rug.nl/ standalone/disclose/

- Supplementary information: http://bioinformat ics.biol.rug.nl/standalone/disclose/

- Operating systems: Microsoft Windows, Linux and Mac OSX

- Programming language: Java

- License: Freely available

- Any restrictions on use by non-academics: No

\section{Authors' contributions}

EJB and SAFTH conceived the study. EJB programmed the program. RS and $\mathrm{KJH}$ designed and programmed the analysis interface. EJ and SAFTH drafted the manuscript. JBTMR and OPK guided and coordinated the project and were involved in correcting and improving the manuscript. All authors read and approved the final manuscript.

\section{Additional material}

\section{Additional file 1}

Validated results of overrepresented DNA binding sites. Various motifs that were identified by the DNA binding site identification module from DISCLOSE matched known motifs described in the literature. The motifs identified by DISCLOSE are visualized as sequence logos [16] and are displayed in the first column. The name of the matching regulon and the sequence logo based on aligned known motif instances are placed in the second and third column respectively.

Click here for file

[http://www.biomedcentral.com/content/supplementary/1471-

2105-9-535-S1.png] 


\section{Acknowledgements}

This study was supported by a grant from the Netherlands Organisation for Scientific Research and industrial partners in the NWO-BMI project number 050.50 .206 on Computational Genomics of Prokaryotes and by Center IOP Genomics. This work was in part supported by an EU program in FW6: Bacell Health, European Union Grant LSHG-CT-2004-503468.

\section{References}

I. Quackenbush J: Computational analysis of microarray data. Nat Rev Genet 200I, 2(6):4l8-427.

2. Eisen MB, Spellman PT, Brown PO, Botstein D: Cluster analysis and display of genome-wide expression patterns. Proc Natl Acad Sci USA 1998, 95(25): |4863-|4868.

3. Toronen P: Selection of informative clusters from hierarchical cluster tree with gene classes. BMC Bioinformatics 2004, 5:32.

4. Gat-Viks I, Sharan R, Shamir R: Scoring clustering solutions by their biological relevance. Bioinformatics 2003, 9(I 8):238I-2389.

5. Gibbons FD, Roth FP: Judging the quality of gene expressionbased clustering methods using gene annotation. Genome Res 2002, I 2(10): | 574-158|.

6. Gasch AP, Eisen MB: Exploring the conditional coregulation of yeast gene expression through fuzzy k-means clustering. Genome Biol 2002, 3( I I):RESEARCH0059.

7. Shamir R, Maron-Katz A, Tanay A, Linhart C, Steinfeld I, Sharan R, Shiloh $Y$, Elkon R: EXPANDER-an integrative program suite for microarray data analysis. BMC Bioinformatics 2005, 6:232.

8. Kim TM, Chung YJ, Rhyu MG, Jung MH: Inferring biological functions and associated transcriptional regulators using gene set expression coherence analysis. BMC Bioinformatics 2007, 8:453.

9. Datta S, Datta S: Methods for evaluating clustering algorithms for gene expression data using a reference set of functional classes. BMC Bioinformatics 2006, 7:397.

10. Jakt LM, Cao L, Cheah KS, Smith DK: Assessing clusters and motifs from gene expression data. Genome Res 200I, I I:I I 2-123.

II. Thijs G, Moreau Y, Smet FD, Mathys J, Lescot M, Rombauts S, Rouze $\mathrm{P}$, Moor BD, Marchal K: INCLUSive: integrated clustering, upstream sequence retrieval and motif sampling. Bioinformatics 2002, I8(2):331-332

12. Chakravarty A, Carlson J, Khetani R, Gross R: A novel ensemble learning method for de novo computational identification of DNA binding sites. BMC Bioinformatics 2007, 8:249.

13. Blom EJ, Bosman DWJ, van Hijum SAFT, Breitling R, Tijsma L, Silvis R, Roerdink JBTM, Kuipers OP: FIVA: Functional Information Viewer and Analyzer extracting biological knowledge from transcriptome data of prokaryotes. Bioinformatics 2007, 23(9): II6I-1163.

14. Makita Y, Nakao M, Ogasawara N, Nakai K: DBTBS: database of transcriptional regulation in Bacillus subtilis and its contribution to comparative genomics. Nucleic Acids Res 2004:D75-D77.

I5. Salgado H, Gama-Castro S, Peralta-Gil M, Díaz-Peredo E, SánchezSolano F, Santos-Zavaleta A, Martínez-Flores I, Jiménez-Jacinto V, Bonavides-Martínez C, Segura-Salazar J, Martínez-Antonio A, Collado-Vides J: RegulonDB (version 5.0): Escherichia coli K-I 2 transcriptional regulatory network, operon organization, and growth conditions. Nucleic Acids Res 2006:D394-D397.

16. Crooks GE, Hon G, Chandonia JM, Brenner SE: WebLogo: a sequence logo generator. Genome Res 2004, | 4(6): I | 88- I 190.

17. Keijser BJF, Beek AT, Rauwerda H, Schuren F, Montijn R, Spek H van der, Brul S: Analysis of temporal gene expression during Bacillus subtilis spore germination and outgrowth. J Bacteriol 2007, I 89(9):3624-3634.

18. NCBI GEO [http://www.ncbi.nlm.nih.gov/geo/]

19. Scheer M, Klawonn F, Münch R, Grote A, Hiller K, Choi C, Koch I, Schobert M, Härtig E, Klages U, Jahn D: JProGO: a novel tool for the functional interpretation of prokaryotic microarray data using Gene Ontology information. Nucleic Acids Res 2006:W510-W515.

20. EBI Genome Reviews [http://www.ebi.ac.uk/GenomeReviews/ files/cellular/]

21. COG WHOG [ftp://ftp.ncbi.nih.gov/pub/COG/COG/whog]
22. KEGG Pathways [ftp://ftp.expasy.org/databases/uniprot/ current release/knowledgebase/taxonomic divisions/ uniprot sprot bacteria.dat.gz]

23. Gene Ontology Obo File [http://www.geneontology.org/ontol ogy/gene ontology.obo]

24. Uniprot [http://www.genome.jp/kegg/catalog/org list.html]
Publish with Bio Med Central and every scientist can read your work free of charge

"BioMed Central will be the most significant development for disseminating the results of biomedical research in our lifetime. "

Sir Paul Nurse, Cancer Research UK

Your research papers will be:

- available free of charge to the entire biomedical community

- peer reviewed and published immediately upon acceptance

- cited in PubMed and archived on PubMed Central

- yours - you keep the copyright

Submit your manuscript here:

http://www.biomedcentral.com/info/publishing_adv.asp
BioMedcentral 